\title{
Calidad de agua en términos físico-químico-metales en tres sitios contrastantes del río Conchos en Chihuahua, México
}

\author{
Water quality using physical-chemical and metal parameters \\ en three contrasting locations of the Conchos river in Chihuahua, Mexico
}

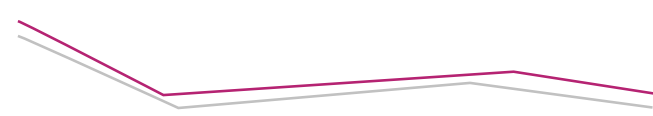

Héctor Rubio Arias ${ }^{1 *}$, Jesús Manuel Ochoa Rivero², Roberto Carlos Ortiz Delgado3', Rey Manuel Quintana', Rubén Alfonso Saucedo Terán², María de Lourdes Villalba ${ }^{4}$

Rubio Arias, H., Ochoa Rivero, J. M., Ortiz Delgado, R. C., Quintana R. M., Saucedo Terán, R. A., Villalba, M. L. Calidad de agua en términos físico-químico-metales en tres sitios contrastantes del río Conchos en Chihuahua, México. Investigación y Ciencia de la Universidad Autónoma de Aguascalientes. Número 70: 13-22, enero-abril 2017

\section{RESUMEN}

En el estado de Chihuahua predominan los ecosistemas áridos y semiáridos, por esta razón, el recurso agua es considerado como primordial. El objetivo fue determinar la calidad del agua en términos físico-químico-metales en tres sitios contrastantes del río Conchos: antes (escorrentía), en la presa (almacenada) y después (liberada). Se colectaron un total de 80 muestras aleatorias de agua; 30 en la localidad de Valle de Zaragoza (VZ), 20 en la presa La Boquilla (PB) y 30 en la localidad de San Francisco de Conchos (SFC). Se cuantificaron los parámetros de temperatura (T), sólidos disueltos totales (SDT), potencial hidrógeno $(\mathrm{pH})$, conductividad eléctrica (CE) y los siguientes metales y metaloides: $\mathrm{Al}, \mathrm{As}, \mathrm{B}, \mathrm{Cd}, \mathrm{Pb}, \mathrm{Se}, \mathrm{Ca}, \mathrm{Cr}$, $\mathrm{Cu}, \mathrm{Co}, \mathrm{Fe}, \mathrm{K}, \mathrm{Mg}, \mathrm{Mn}, \mathrm{Na}, \mathrm{Ni}, \mathrm{Si}$ y $\mathrm{Zn}$.

Se realizó un análisis de varianza (ANOVA) y la prueba de Tukey para la comparación de medias y se consideró un nivel de significancia de 0.05 ( $\alpha=$

Palabras clave: calidad agua, río Conchos, Chihuahua, México, metales.

Keywords: water quality, Conchos river, Chihuahua, Mexico, metals.

\section{Recibido: 14 de agosto de 2015, aceptado: 1 de julio de 2016}

Facultad de Zootecnia y Ecología, Universidad Autónoma de Chihuahua, México.

Instituto Nacional de Investigaciones Forestales, Agrícolas y Pecuarias sitio experimental La Campana, México.

Consultoría independiente.

4 Facultad de Ingeniería, Universidad Autónoma de Chihuahua, México.

* Autor para correspondencia: rubioa1105@hotmail.com
0.05). Los resultados no mostraron diferencias para $\mathrm{pH}(\mathrm{VZ}=8.53 ; \mathrm{PB}=8.60 ; \mathrm{SFC}=8.42)$ pero sí para $\mathrm{T}(\mathrm{VZ}=$ $\left.17.5^{\circ} \mathrm{C} ; \mathrm{PB}=14.2^{\circ} \mathrm{C} ; \mathrm{SFC}=15.2^{\circ} \mathrm{C}\right), \mathrm{CE}(\mathrm{VZ}=0.33 \mu \mathrm{S}$ $\mathrm{cm}^{-2} ; \mathrm{PB}=0.18 \mu \mathrm{S} \mathrm{cm}-2 ; \mathrm{SFC}=0.18 \mu \mathrm{S} \mathrm{cm} \mathrm{cm}^{-2}$ ) y SDT (VZ= $\left.273.90 \mathrm{mg} / \mathrm{l}^{-1} \mathrm{~PB}=0.33 \mathrm{mg} / \mathrm{l}^{-1} ; \mathrm{SFC}=259.8 \mathrm{mg} / \mathrm{l}^{-1}\right)$. Los elementos As, Ag, Cd no fueron detectados y la concentración de $\mathrm{Al}, \mathrm{B}, \mathrm{Ca}, \mathrm{Cr}$ y $\mathrm{Mg}$ fue diferente entre sitios, pero no para $\mathrm{Mn}, \mathrm{Na}, \mathrm{Pb}, \mathrm{Se}, \mathrm{Cu}, \mathrm{Fe}, \mathrm{K}$, $\mathrm{Ni}$, Si y Zn. Se concluye que el agua bajo estudio es segura con fines agropecuarios y desde el punto de vista ecológico.

ABSTRACT

The state of Chihuahua is located in a geographic region where half of the State is considered to be a semiarid or arid environment; for this reason, the water resource is a fundamental necessity. The objective was to determine the water quality in terms of physical-chemical-metals parameters at three location points of the Conchos river; before being captioned in the dam and after being captioned. A total of 80 random samples were collected; thirty randomly water samples were collected in Valle de Zaragoza (VZ), 20 samples in the Boquilla dam (PB) and 30 samples in San Francisco de Conchos (SFC). The measured parameters were temperature (T), total dissolved solids (TDS), hydrogen potential (pH), electrical conductivity (EC) and the following metals and metalloids; Al, As, B, Cd, Pb, Se, Ca, Cr, Cu, Co, $\mathrm{Fe}, \mathrm{K}, \mathrm{Mg}, \mathrm{Mn}, \mathrm{Na}, \mathrm{Ni}, \mathrm{Si}$ y $\mathrm{Zn}$. An analysis of variance (ANOVA) was conducted for each parameter and when statistical difference between locations was found, the Tukey's test was performed. In all cases 
IIVESTIGACIÓn Y CIERCIA DE LA UחIVERSIDAD AUTÓnOMH DE RGUASCALIERTES a significance level of 0.05 was considered ( $\alpha=0.05)$. The results did not show statistical differences for $\mathrm{pH}$ $(\mathrm{VZ}=8.53 ; \mathrm{PB}=8.60 ; \mathrm{SFC}=8.42)$ but differences were noted for $\mathrm{T}\left(\mathrm{VZ}=17.5^{\circ} \mathrm{C} ; \mathrm{PB}=14.2^{\circ} \mathrm{C}\right.$; $\left.\mathrm{SFC}=15.2^{\circ} \mathrm{C}\right)$, $E C\left(V Z=0.33 \mu S \mathrm{~cm}^{-2} ; \mathrm{PB}=0.18 \mu \mathrm{S} \mathrm{\textrm {cm } ^ { - 2 }} ; \mathrm{SFC}=0.18 \mu \mathrm{S}\right.$ $\left.\mathrm{cm}^{-2}\right)$ and TDS $\left(\mathrm{VZ}=273.90 \mathrm{mg} / \mathrm{l}^{-1} ; \mathrm{PB}=0.33 \mathrm{mg} / \mathrm{l}^{-1} ; \mathrm{SFC}=\right.$ $\left.259.8 \mathrm{mg} / \mathrm{l}^{-1}\right)$. The elements $\mathrm{As}, \mathrm{Ag}$, and $\mathrm{Cd}$ were not detected and the concentration for $\mathrm{Al}, \mathrm{B}, \mathrm{Ca}, \mathrm{Cr}$, and $\mathrm{Mg}$ was different due to locations but not for $\mathrm{Mn}$, $\mathrm{Na}, \mathrm{Pb}, \mathrm{Se}, \mathrm{Cu}, \mathrm{Fe}, \mathrm{K}, \mathrm{Ni}, \mathrm{Si}$ and $\mathrm{Zn}$. It is concluded that the water of the study area is safe for irrigation as well as for ecological purposes.

INTRODUCCIÓN

De acuerdo con la ONU (Organización de las Naciones Unidas), el agua es un recurso natural limitado que debe ser considerado como bien público, ya que es fundamental para toda forma de vida $y$, en especial, para la salud humana (ONU, s. f.). Este recurso cubre $71 \%$ de la superficie terrestre y se localiza principalmente en los océanos, donde se concentra $96.5 \%$ del agua. El resto se encuentra en glaciares y casquetes polares que contienen $1.74 \%$, así como en depósitos subterráneos, permafrost (siempre congelados) y glaciares continentales con $1.72 \%$. El restante $0.04 \%$ se reparte entre lagos, humedad en suelo, atmósfera, embalses, ríos y seres vivos (Gleick et al., 2002).

El agua que fluye en el río Conchos es considerada como el recurso natural más importante del norte de México y representa la principal corriente superficial del estado de Chihuahua (CNA, 2010). Este río nace en la vertiente oriental de la Sierra Madre Occidental, en la región conocida como la Alta Sierra Tarahumara. Luego, sus aguas cruzan las amplias llanuras del desierto chihuahuense y, finalmente, se incorporan al río Bravo/río Grande en la zona fronteriza de Ojinaga (Kelly, 2001; Colegio de Chihuahua, 2007). Es importante mencionar que en el estado de Chihuahua existen embalses que han sido construidos por el hombre, como la presa La Boquilla (Lago Toronto), la Francisco I. Madero (Las Vírgenes) y la Luis L. León (El Granero); los cuales captan el agua de los diversos tributarios del río Conchos y la utilizan en forma posterior para diversos propósitos. De toda la infraestructura hidráulica del estado de Chihuahua, la más importante y de mayor magnitud es la presa La Boquilla, que tiene una capacidad de 2,903 $\mathrm{Mm}^{3}$ (INEGI, 2003).
Para la cuenca hidrológica del Conchos, algunos estudios previos han indicado diversos niveles de contaminación del agua que fluye en sus tributarios (Rubio et al., 2004; Holguín et al., 2006; Gutiérrez et al., 2008; Rubio Arias et al., 2012), de su área ribereña (Rubio Arias et al., 2005) e incluso, en centros recreativos o de esparcimiento, como es el caso del Lago Colina (Rubio Arias et al., 2011). Este efecto de contaminación incluye los principales embalses de Chihuahua que presentan niveles considerables en diversos parámetros, en especial de metales pesados; por ejemplo, Hernández et al. (2007) encontraron concentraciones de $\mathrm{Fe}$, $\mathrm{Ni}$ y $\mathrm{Pb}$ que rebasaron los niveles máximos permitidos por la NOM-001-ECOL-1996 en tres presas del estado. Debido a la importancia de este recurso natural, es imprescindible determinar las condiciones del agua que fluye en el río Conchos y, a su vez, determinar los posibles cambios que se puedan presentar durante el tiempo de almacenamiento. Además, es necesario identificar los potenciales efectos antropológicos como la concentración de metales pesados o algún otro elemento que pudiera estar afectando al medio ambiente, a su flora y fauna, así como a los habitantes establecidos en los márgenes, ya que de esta zona se extrae gran parte del pescado que se consume en la región.

El objetivo de este estudio fue cuantificar el nivel de contaminación del agua del río Conchos en términos físico-químico-metales antes y después de ser almacenada, así como durante su almacenamiento en la presa La Boquilla. Se espera que estos resultados sirvan a las autoridades de los diferentes niveles de gobierno, así como a los habitantes locales a conocer el grado de contaminación, con el fin de proponer esquemas futuros de manejo y de remediación, en su caso.

\section{MATERIALES Y MÉTODOS}

\section{Descripción y localización del área de estudio}

El río Conchos es considerado el más caudaloso e importante del estado de Chihuahua, y el principal afluente del río Bravo/río Grande que sirve como barrera natural entre Estados Unidos y México. La cuenca hidrológica del Conchos tiene una extensión de $68,606 \mathrm{~km}^{2}$ y se encuentra en su mayoría dentro del estado de Chihuahua, cubre alrededor de $26 \%$ de su superficie. Una de las principales funciones de esta cuenca hidrológica es la de abastecer de agua a miles de habitantes, quienes la aprovechan 
por medio de la construcción de represas o grandes presas de almacenamiento. Antes de ser captada en la presa La Boquilla, las aguas del río Conchos pasan cerca de la comunidad Valle de Zaragoza, que se localiza a $27^{\circ} 27^{\prime} 22^{\prime \prime}$ norte, y a 105\%48'39" oeste, a 1,340 m.s.n.m. (Figura 1). En esta localidad se presenta un clima semiárido extremoso con una temperatura máxima extrema de $41.0^{\circ} \mathrm{C}$, y una media mínima de $-5.5^{\circ} \mathrm{C}$, media anual de $18.7^{\circ} \mathrm{C}$. La precipitación pluvial media anual es de $466.1 \mathrm{~mm}$, con vientos dominantes del suroeste (INEGI, 2005). La presa La Boquilla se construyó en 1915 (Dean y Schmidt, 2011) con el propósito de almacenar el agua proveniente del río Conchos, generar energía y ser utilizada en actividades de irrigación. Este reservorio está localizado en el municipio de San Francisco de Conchos, el cual se encuentra a 250 $\mathrm{km}$ de la capital del estado (Gutiérrez y Borrego, 1999). Luego de su almacenamiento, el agua es liberada cerca de la comunidad La Boquilla, la cual se encuentra en el municipio de San Francisco de

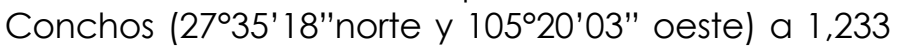
m.s.n.m. (Figura 1). En esta región el clima es semiárido extremoso, con una temperatura máxima de $41.7^{\circ} \mathrm{C}$, mínima de $14.1^{\circ} \mathrm{C}$; precipitación pluvial media anual de $363.9 \mathrm{~mm}$ y los vientos dominantes provienen del suroeste (INEGI, 1998).

\section{Obtención de muestras de agua}

Se realizaron tres muestreos durante el mes de marzo de 2010. El primero se realizó en el río Conchos, cerca de la localidad de Valle de Zaragoza (VZ); es decir, antes de que las aguas del río fueran captadas en la presa La Boquilla (PB). En la sección de VZ se

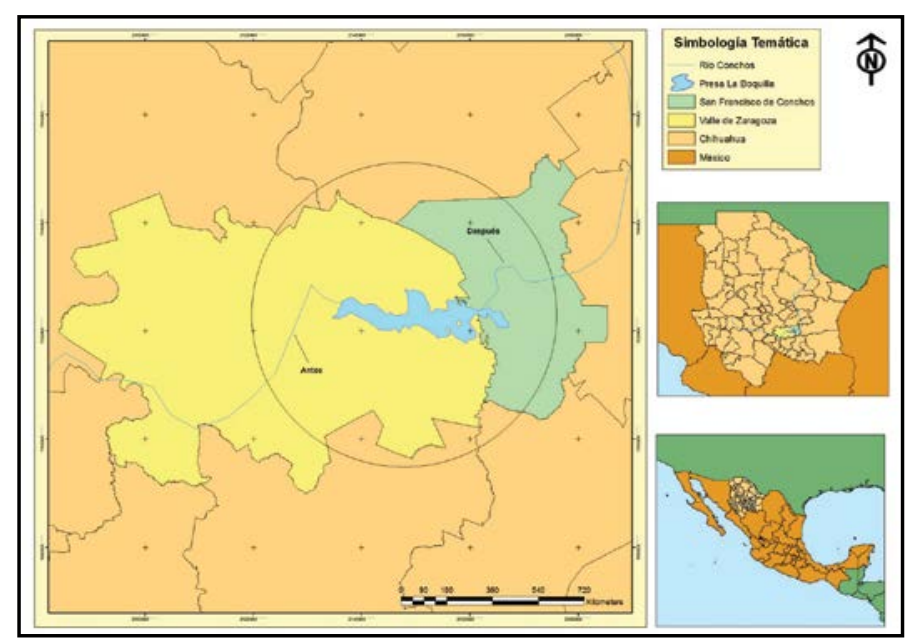

Figura 1. Ubicación geográfica de los tres sitios de muestreo en el río Conchos en Chihuahua, México. Elaboración propia. obtuvieron 30 muestras aleatorias en un tramo de $1,000 \mathrm{~m}$ a una profundidad de $0.30 \mathrm{~m}$. Las muestras fueron colectadas en sentido contrario a la corriente en envases previamente lavados y etiquetados con capacidad de 1 I. Cada sitio fue debidamente georreferenciado con ayuda de un GPS comercial (Garmin-TM). El segundo muestreo se realizó en la presa La Boquilla, para lo cual se utilizó una lancha con motor fuera de borda. En forma previa se cuadriculó el área total de almacenamiento y luego se seleccionaron 20 cuadrantes de manera aleatoria. En cada uno se recolectó una muestra de agua a los $0.30 \mathrm{~m}$ de profundidad. Al igual que en el primer muestreo los recipientes fueron lavados, etiquetados y cada punto georreferenciado. El tercer muestreo se realizó después de que las aguas fueran liberadas de la presa en un lugar cercano a la comunidad de San Francisco de Conchos (SFC), donde se localizaron puntos aleatorios de la misma forma en que se realizó en el tramo de la localidad de Valle de Zaragoza, se colectaron 30 muestras de agua. Se obtuvieron un total de 80 muestras; 30 en Zaragoza (VZ); 20 en la presa La Boquilla (PB) y 30 en San Francisco de Conchos (SFC). Las mismas fueron colocadas en hieleras para su debida conservación a una temperatura aproximada de $4^{\circ} \mathrm{C}$ y trasladadas al Laboratorio de Parámetros Ambientales de la Facultad de Zootecnia y Ecología de la Universidad Autónoma de Chihuahua para su posterior análisis. La toma de muestras se efectuó de acuerdo al criterio citado en la Norma mexicana NMX-AA-014-1980 (SCFI, 1980) que especifica sobre los lineamientos y recomendaciones para muestreos en cuerpos receptores de aguas superficiales.

\section{Análisis de las muestras}

El parámetro de temperatura (T) se obtuvo in situ con un termómetro de mercurio de acuerdo con la Norma mexicana NMX-AA-007-SCFI-2000. Los parámetros de $\mathrm{pH}$ y de conductividad eléctrica (CE) se cuantificaron también in situ utilizando un potenciómetroconductivímetro marca Hanna Instruments ${ }^{\top \mathrm{M}}$ Modelo Waterproof $\mathrm{pH} / \mathrm{CE} / \mathrm{Temp}$ de acuerdo con las normas NMX-AA-008-SCFI-2000 y NOM-AA-093-SCFI-2000. La cuantificación de Ag, Al, As, B, Ca, Cd, Cr, Cu, $\mathrm{Fe}, \mathrm{K}, \mathrm{Mg}, \mathrm{Mn}, \mathrm{Na}, \mathrm{Ni}, \mathrm{Pb}, \mathrm{Se}$, Si y $\mathrm{Zn}$ se realizó en un espectrofotómetro de emisión óptica por plasma acoplado inductivamente (ICP-OES) 2100 Perkin ElmerTM. Previo a la cuantificación de metales a las muestras se les realizó una digestión de acuerdo con la Norma mexicana NMX-AA-051-SCFI-2001. 
IIVESTIGACIÓn Y CIERCIA DE LA UNIVERSIDAD AUTÓNOMA

\section{Análisis estadístico}

Se realizó un análisis de varianza (ANOVA) para detectar diferencias entre los tres puntos de muestreo y cuando se encontraron diferencias estadísticas entre sitios se utilizó la herramienta estadística de comparación de medias empleando la prueba de Tukey (Rubio y Jiménez, 2012). En todos los casos se consideró un nivel de significancia de $0.05(\alpha=0.05)$.

\section{RESULTADOS}

No se detectaron diferencias entre sitios para el parámetro de $\mathrm{pH}(P>0.05)$. La media en VZ fue 8.53, mientras que en PB fue de 8.60 y de 8.42 en SFC. La concentración de CE fue diferente entre sitios $(P<0.05)$ y se detectó que la media en $V Z$ tuvo un valor de $0.34 \mu \mathrm{S} \mathrm{cm}^{-2}$, distinto a los obtenidos en $\mathrm{PB}$ y en SFC, que presentaron valores de $0.18 \mu \mathrm{S} \mathrm{cm}^{-2}$ (Figura 2a). Para la variable T, el ANOVA detectó diferencias entre sitios $(P<0.05)$, donde la media en VZ tuvo un valor de $17.5^{\circ} \mathrm{C}$ y fue estadísticamente diferente a los valores de PB con $14.2^{\circ} \mathrm{C}$ y a los de SFC con $15.2^{\circ} \mathrm{C}$ (Figura 2b). Para SDT, el ANOVA detectó diferencias entre sitios $(P<0.05)$, con valor diferente la media en VZ con $273.90 \mathrm{mg} \mathrm{l}^{-1}$ de la de PB con 0.33 $\mathrm{mg} \mathrm{l}^{-1}$, pero igual al valor en SFC con $259.8 \mathrm{mg} \mathrm{l}^{-1}$.
Los siguientes elementos no fueron encontrados en ninguna de las muestras; Ag, As y Cd, o bien, fueron ligeramente detectados. En especial los resultados para As estuvieron por abajo de $0.01 \mathrm{mg} \mathrm{t}^{-1}$ y fueron inconsistentes, por lo cual no se discuten los resultados de este metaloide ni tampoco los de Ag y Cd. El As es difícil de cuantificar y en ocasiones su rango de lectura presenta altos contrastes debido a que son pequeñas cantidades y su varianza, en consecuencia, es alta. Para la concentración de Al, el ANOVA detectó diferencias entre sitios de muestreo $(P<0.05)$ donde la media en VZ fue de $0.28 \mathrm{mg} \mathrm{t}^{-1}$, mientras que en PB y SFC se encontraron niveles similares $10.76 \mathrm{mg} \mathrm{t}^{-1} \mathrm{y}$ de $0.77 \mathrm{mg}^{-1}$ ) que se muestran en la Figura $2 \mathrm{~d}$. La prueba de Tukey encontró que la concentración en VZ fue diferente a las otras dos localidades, mientras que los niveles en PB y SFC fueron estadísticamente similares. Para el elemento $B$ se encontraron diferencias entre zonas de muestreo $(P<0.05)$, donde la media en $V Z$ fue de $0.11 \mathrm{mg} \mathrm{l}^{-1}$, mientras que en PB fue de $0.14 \mathrm{mg} \mathrm{t}^{-1}$ y de $0.03 \mathrm{mg} \mathrm{l}^{-1}$ en SFC. La prueba de Tukey demostró una igualdad estadística entre VZ y PB, pero diferente a SFC. La concentración de Ca fue diferente para las zonas de muestreo $(P<0.05)$. La mayor concentración se detectó en $\mathrm{VZ}$ con $54.10 \mathrm{mg} \mathrm{l}^{-1}$, mientras que en PB fue de $48.36 \mathrm{mg} \mathrm{l}^{-1}$ y luego disminuyó en SFC con 34.15

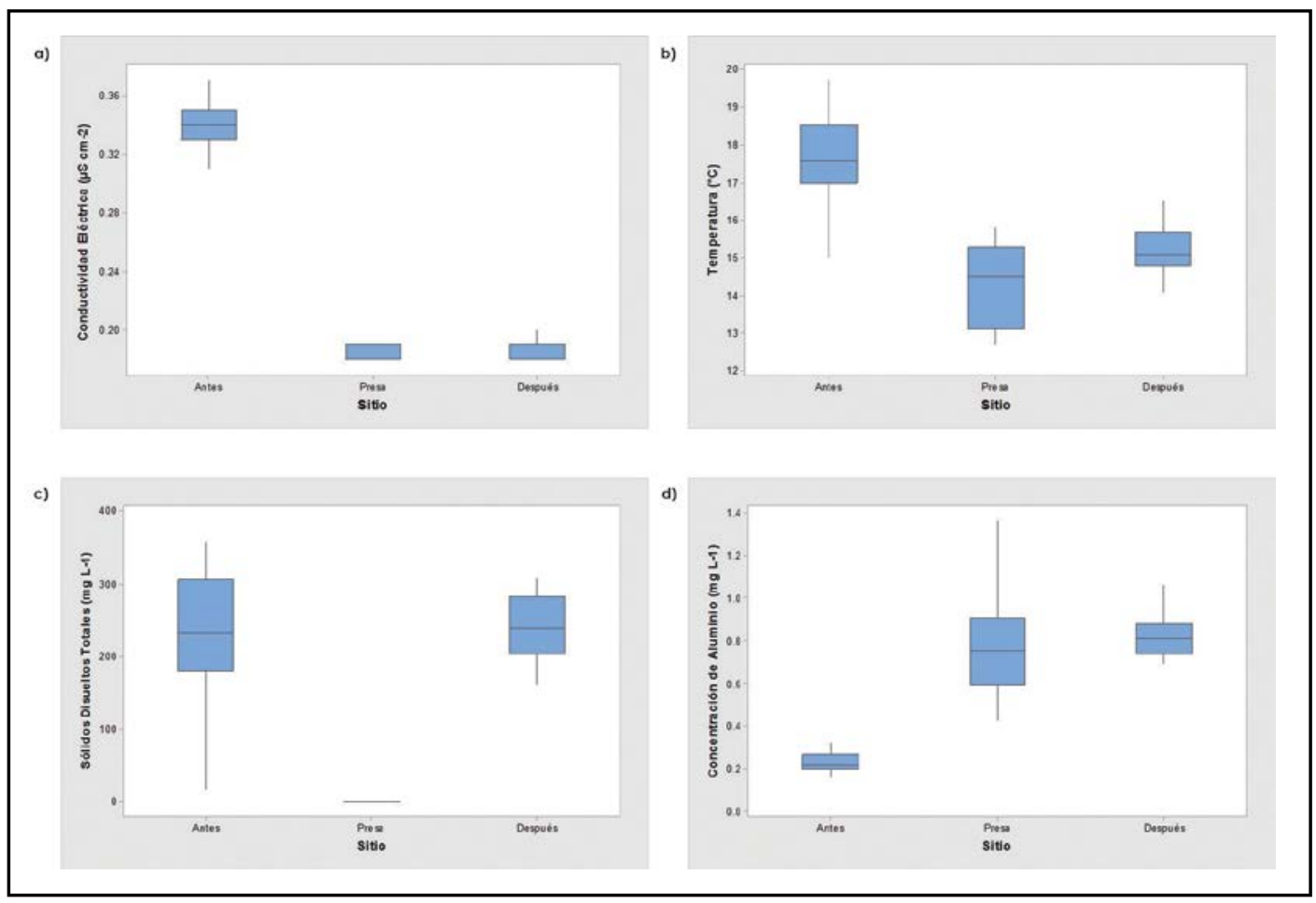

Figura 2. Concentración de las variables conductividad eléctrica (a), temperatura (b), sólidos disueltos totales (c) y Aluminio (Al) en agua del río Conchos. Elaboración propia. 


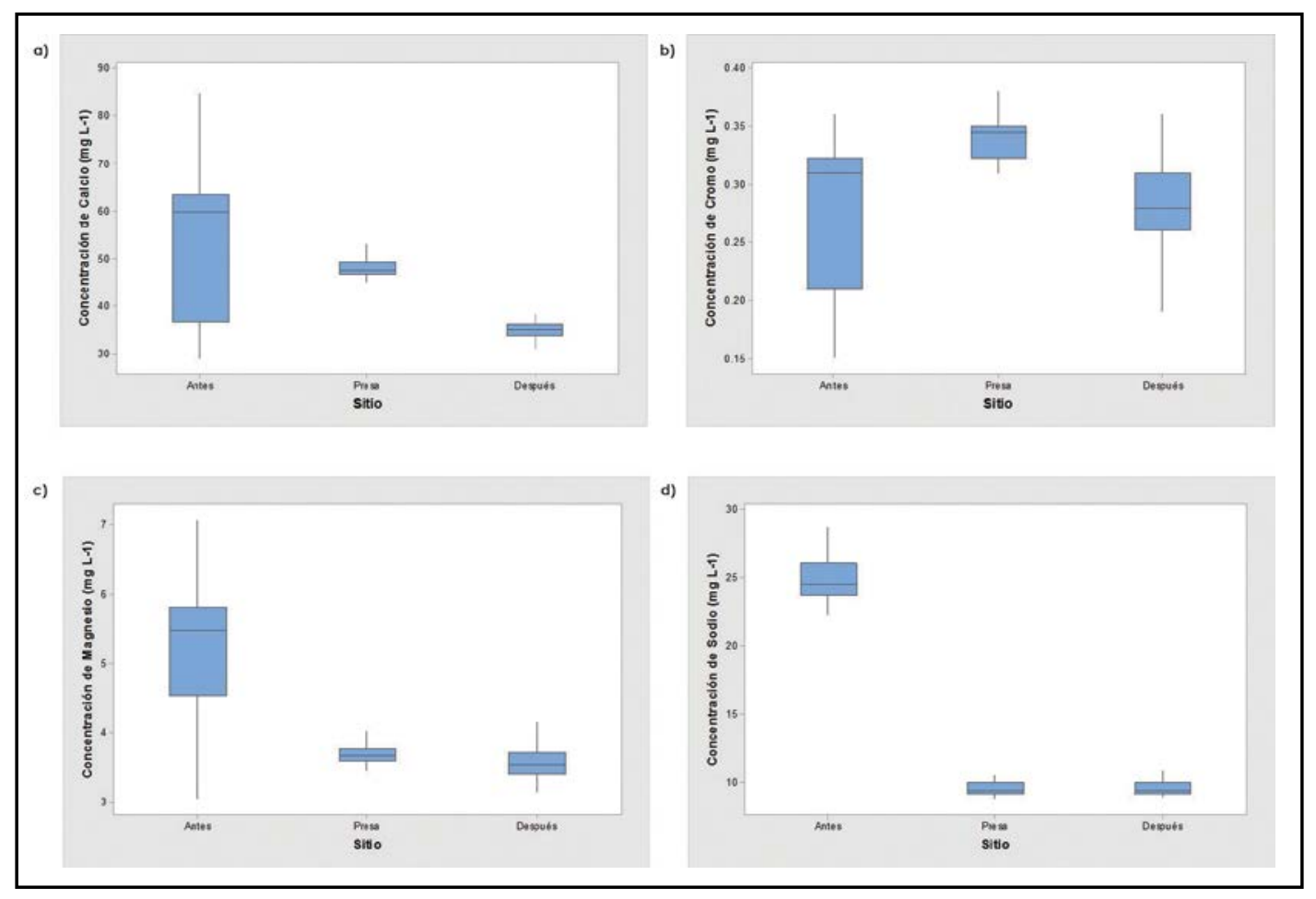

Figura 3. Concentración de las variables calcio (a), cromo (b), magnesio (c) y sodio (d) en agua del río Conchos. Elaboración propia.

$\mathrm{mg} \mathrm{l}^{-1}$ (Figura 3a). Los niveles de Cr fueron diferentes entre zonas de muestreo $(P<0.05)$. La concentración media en $\mathrm{VZ}$ fue de $0.27 \mathrm{mg}^{1-1}$ y este valor fue diferente estadísticamente a la concentración en PB $10.37 \mathrm{mg}$ $\left.\mathrm{I}^{-1}\right)$, pero no diferente de SFC $\left(0.27 \mathrm{mg}^{-1}\right)$.

No se encontraron diferencias estadísticas entre zonas de muestreo para $\mathrm{Cu}, \mathrm{Fe}, \mathrm{K}, \mathrm{Ni}, \mathrm{Si}$ y $\mathrm{Zn}$. Los niveles de Cu para VZ y SFC fueron de $0.06 \mathrm{mg}^{-1} \mathrm{y}$ de $0.07 \mathrm{mg}^{-1}$ en PB. Para el caso de Fe se detectó una concentración de $0.83 \mathrm{mg} \mathrm{l}^{-1}$ para VZ y SFC, esta concentración se incrementó ligeramente en PB con $0.85 \mathrm{mg} \mathrm{l}^{-1}$. El K tuvo una concentración de $5.68 \mathrm{mg} \mathrm{t}^{-1}$ en VZ, de $5.39 \mathrm{mg} \mathrm{l}^{-1}$ en PB y de $5.82 \mathrm{mg} \mathrm{l}^{-1}$ en SFC. Para $\mathrm{Ni}$, la mayor concentración se notó en $\mathrm{VZ}$ con $1.37 \mathrm{mg}$ $\mathrm{H}^{-1}$ y la menor en SFC con $1.28 \mathrm{mg} \mathrm{l}^{-1}$. El nivel de Si fue similar estadísticamente con valores de $7.78 \mathrm{mg} \mathrm{t}^{-1} \mathrm{en}$ $\mathrm{VZ}$, de $7.72 \mathrm{mg} \mathrm{l}^{-1}$ en PB y de $7.36 \mathrm{mg} \mathrm{l}^{-1}$ en SFC. Para el Zn la mayor concentración se observó en VZ con $0.117 \mathrm{mg} \mathrm{L}^{-1}$, después PB con $0.10 \mathrm{mg} \mathrm{l}^{-1} \mathrm{y}$ con $0.07 \mathrm{mg}$ $1^{-1}$ en SFC.

Para el caso particular de Mg se encontraron diferencias entre zonas de muestreo $(p<0.05)$ detectando la mayor concentración en VZ con $5.24 \mathrm{mg} \mathrm{l}^{-1}$ y siendo estadísticamente diferente para PB con $3.70 \mathrm{mg} \mathrm{l}^{-1}$ y para SFC con $3.50 \mathrm{mg} \mathrm{l}^{-1}$; estas dos últimas medias fueron estadísticamente iguales (Figura 3c). El nivel de Mn no fue diferente entre sitios $(P<0.05)$. La media en VZ fue de $0.041 \mathrm{mg} \mathrm{L}^{-1}$, en SFC de $0.045 \mathrm{mg} \mathrm{l}^{-1}$ y la concentración en PB fue de 0.050 $\mathrm{mg} \mathrm{l}^{-1}$. El Na fue diferente entre puntos de muestreo $(P<0.05)$. La mayor concentración se observó en VZ con $23.58 \mathrm{mg} \mathrm{l}^{-1}$ y la menor fue en PB con $9.54 \mathrm{mg}$ $\mathrm{I}^{-1}$, mientras que en SFC la concentración fue de $10.20 \mathrm{mg} \mathrm{l}^{-1}$. El Pb fue diferente entre sitios $(P<0.05)$. La mayor concentración se observó en SFC con 0.045 $\mathrm{mg} \mathrm{l}^{-1}$ y la menor en PB con $0.025 \mathrm{mg} \mathrm{l}^{-1}$, por lo que se nota un nivel de $0.034 \mathrm{mg} \mathrm{l}^{-1}$ en VZ. Finalmente, el Se no fue diferente entre localidades de muestreo con niveles de alrededor de $0.012 \mathrm{mg} \mathrm{L}^{-1}$ en los tres sitios.

\section{DISCUSIÓN}

Los altos valores de $\mathrm{pH}$ observados en VZ y en PB, posiblemente se deban a la presencia de sales disueltas y materiales suspendidos que transporta el agua en su trayectoria de las partes altas hacia las bajas (Mosley, 2015). El nivel de pH está relacionado con estrés, mortalidad o diversas enfermedades en un determinado ecosistema acuático (Sansawat y Thirabunyanon, 2009; Melgar Valdés et al., 2013). Los valores de $\mathrm{pH}$ observados en esta investigación concuerdan con los resultados de estudios previos 


\section{INVESTIGAGIÓก Y CIECEIA DE LA UחIVERSIDAD AUTÓกOMA DE RGUASCALIERTES}

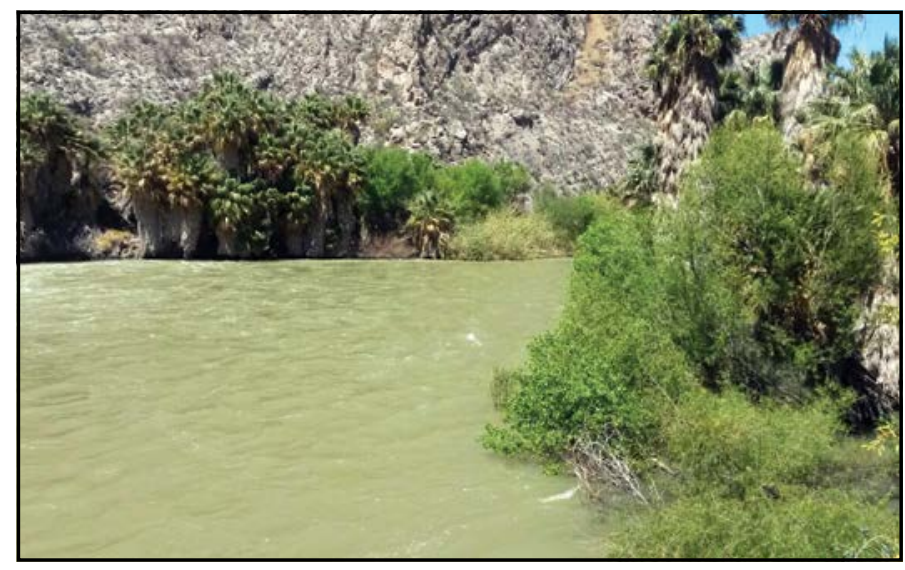

Figura 4. Parte de la zona en estudio. Fotografía tomada por el equipo de investigación.

realizados en diferentes ecosistemas acuáticos de la zona (Holguín et al., 2006; Gutiérrez et al., 2008; Rubio Arias et al., 2011; Rubio Arias et al., 2012). Con respecto a los niveles de $C E$, la mayor concentración en VZ se puede explicar por la disolución del material geológico presente en la zona (Miyamoto et al., 1995) y un efecto de dilución al momento de llegar a la presa La Boquilla. Los resultados encontrados en este estudio son menores a los reportados por otros investigadores en diferentes segmentos del río Conchos. Por ejemplo, Gutiérrez y Carreón Hernández (2004) notaron niveles tan altos como $2,080 \mu \mathrm{S} \mathrm{cm}^{-2}$ en un punto que recibía descargas urbanas y agrícolas, pero que disminuían hasta 1,050 $\mu S \mathrm{~cm}^{-2}$ al llegar a un centro receptor (presa Luis L. León).

La T detectada en los tres sitios concordó con las condiciones climáticas de la temporada invernal en la región. No obstante, el valor en el agua de la presa se debió a que la toma de muestras se realizó a horas del amanecer, lo que ocasionó una variación con respecto a los demás sitios de muestreo. Preece y Jones (2002) mencionaron que el agua a nivel superficial se vuelve más densa en el período invernal, lo que ocasiona temperaturas bajas en el recurso hídrico. Sin embargo, estos cambios también se pueden atribuir a que en los ecosistemas acuáticos, esta variable se rige con base en la temperatura ambiente, flujos de agua (entrada/salida) y el tiempo que dura el agua en la presa (Arbat Bofill et al., 2014). Los investigadores Webb et al. (2008) señalaron que el agua que es liberada de una presa suele ser más fría que dentro y fuera del cuerpo de almacenamiento debido a los cambios térmicos que ocurren por la estratificación

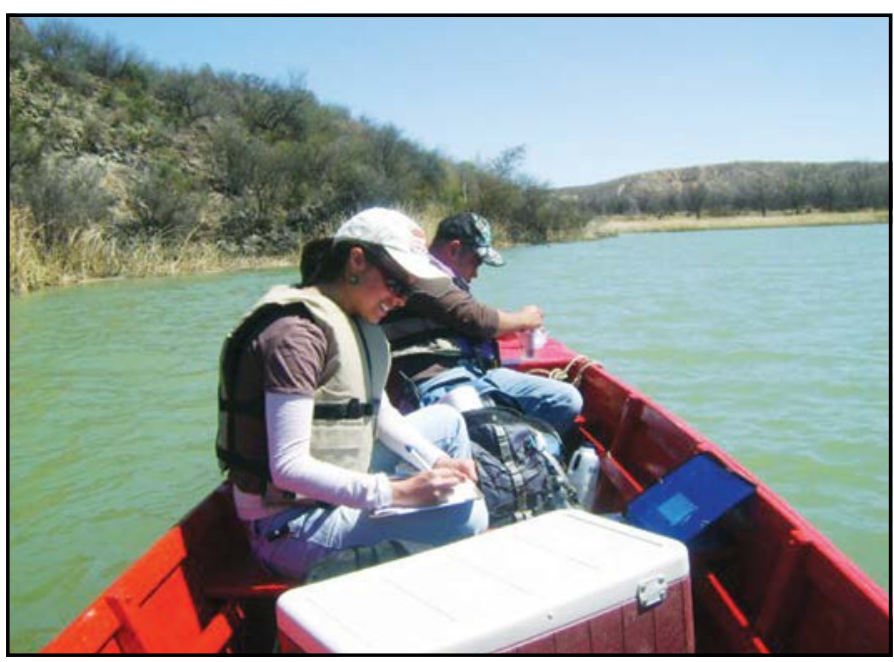

Figura 5. Se estudia la calidad del agua en el río Conchos. Fotografía tomada por el equipo de investigación.

del vital líquido. De esta manera, se corrobora el valor encontrado después de este reservorio. Es importante mencionar que las variaciones en $\mathrm{T}$ tienen una influencia directa sobre la composición química del agua y una correlación directa con el OD (Preece y Jones, 2002; Rubio Arias et al., 2013). Para el parámetro de SDT, el valor más alto que se observó en la localidad de VZ está indicando la presencia de una mayor cantidad de iones solubles en su mayor parte orgánicos, que están siendo trasportados en el caudal, lo que se demostró con la correlación que se detectó entre las variables de SDT y CE $(P<0.05)$. El bajo valor observado en la presa de SDT (Figura 2c) refleja el proceso de sedimentación que ocurre en el ecosistema y la poca velocidad que se presenta en el caudal (Caruso, 2002).

Está bien documentado que la concentración de metales en agua puede representar un serio peligro para la vida acuática. Por ejemplo, el Al puede conllevar un peligro cuando se tiene un $\mathrm{pH}$ menor de 6 (CCREM, 1987), por lo que los resultados de este estudio infieren que este elemento no está afectando la fauna acuática de este ecosistema. No obstante, sin considerar el valor de $\mathrm{pH}$, la concentración de Al potencialmente representa un factor de riesgo para la vida acuática, debido a que los valores observados superan lo establecido en la normatividad CE-CCA-001/89 para agua dulce y protección de vida acuática que es de $0.05 \mathrm{mg} \mathrm{l}^{-1}$ (SEDUE, 1989). Es importante mencionar que el agua del río Conchos puede ser utilizada para el riego agrícola y uso pecuario debido a que los niveles de Al cuantificados se encuentran por debajo 


\section{IIVESTIGAGIÓn Y CUERCIA DE LA UחIVERSIDAD AUTÓNOMA DE AGUASCALIERTES}

de los criterios establecidos por la normatividad mexicana, que es de $5 \mathrm{mg} \mathrm{l}^{-1}$ (SEDUE, 1989; NOM, 1993). Este elemento puede ser movilizado de suelos y sedimentos por el lixiviado natural, por lo que la mayor concentración en SFC puede ser explicado por la movilización natural de este elemento y, tal vez, por una acción antropogénica, ya que el agua de la presa recibe escorrentías suministradas tanto por la cabecera municipal de Valle de Zaragoza como por la zona agrícola establecida en la parte superior de la presa.

Los resultados de este estudio muestran que el nivel de $B$ en los tres sitios no superó los límites establecidos en la Norma Oficial Mexicana CECCA-001/89 de usos del agua, que establece un límite de $1.0 \mathrm{mg} \mathrm{l}^{-1}$ (SEDUE, 1989). Del mismo modo, dichos valores se encuentran por debajo de los niveles permisibles para agua de consumo humano y/o animal, que es $0.5 \mathrm{mg} \mathrm{H}^{-1}$ (Tagliabue et al., 2013; Kochkodan et al., 2015). Es importante mencionar que en un estudio previo en el río Conchos, realizado por Holguín et al. (2006), se reportaron niveles similares al presente estudio, con una media de $0.1944 \mathrm{mg} \mathrm{l}^{-1}$, lo que ejemplifica que las concentraciones de $\mathrm{B}$ en el agua del Conchos han sido constantes con el paso del tiempo. La presencia de B en el agua superficial puede ocurrir de manera natural (geo-hidrología) o por efectos antropogénicos. Por ejemplo, las potenciales fuentes de contaminación de origen humano son la industria minera, la metalurgia y las actividades agropecuarias (Tagliabue et al., 2013; Kochkodan et al., 2015).

Con respecto al elemento Ca, este se encuentra en ciertos tipos de roca como calizas y dolomitas y se considera esencial para el ser humano; donde de hecho, es el elemento más abundante en el cuerpo

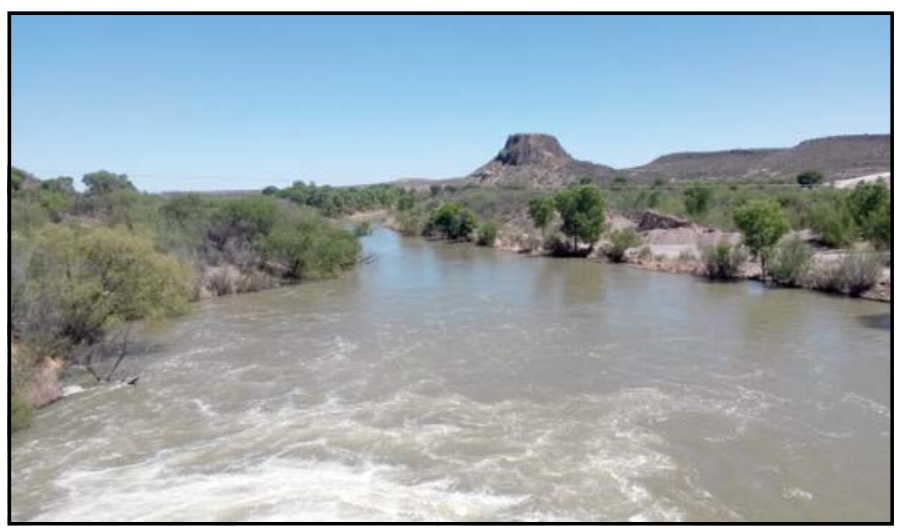

Figura 6. Toma del río Conchos, en Chihuahua, México.

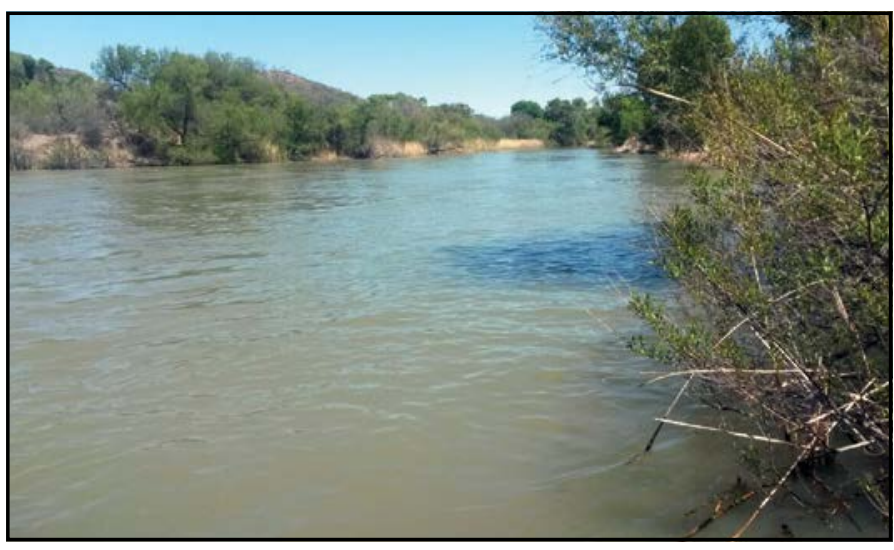

Figura 7. Las concentraciones de $\mathrm{Cr}$ obtenidas permiten inferir que el agua del río Conchos se encuentra dentro de los límites permitidos por la normatividad mexicana para su utilización en el sector agropecuario. Fotografía tomada por el equipo de investigación.

humano. No obstante, un exceso de Ca puede provocar en el hombre el desarrollo de piedrecillas en los riñones o esclerosis y taponamientos de venas y arterias (NIH, 2013). En el caso particular de peces y organismos acuáticos, un exceso de Ca no parece ser perjudicial (Brooks et al., 2003). No existen a la fecha restricciones en alguna normatividad nacional o extranjera para las concentraciones de este elemento, por lo que el agua de esta sección del río Conchos puede ser utilizada en diferentes actividades. Otras investigaciones en la parte baja del río Conchos han detectado mayores valores de Ca con niveles de $137.50 \mathrm{mg} \mathrm{l}^{-1}$ (Gutiérrez et al., 2008), de $202.74 \mathrm{mg} \mathrm{l}^{-1}$ (Holguín et al., 2006) y de $319.0 \mathrm{mg} \mathrm{l}^{-1}$ (Gutiérrez y Carreón Hernández, 2004) en comparación con los reportados en este estudio.

Las concentraciones de $\mathrm{Cr}$ obtenidas permiten inferir que el agua del río Conchos se encuentra dentro de los límites permitidos por la normatividad mexicana para su utilización en el sector agropecuario $\left(1.0 \mathrm{mg} \mathrm{l}^{-1}\right)$ sin límites para la protección de la vida acuática (SEDUE, 1989; NOM, 1993). La mayor concentración en PB (Figura 3b) posiblemente se explique por el arrastre de $\mathrm{Cr}$ de la parte alta de la presa, ya que su presencia se ha relacionado con la aplicación de fertilizantes y pesticidas (Charef et al., 2011). Con respecto a $\mathrm{Mg}$, a la fecha no existen restricciones para sus niveles, incluyendo el suministro de agua potable, por lo que el agua se puede considerar como segura para cualquier uso. Otros estudios en la cuenca hidrológica han reportado niveles de $16.10 \mathrm{mg} \mathrm{l}^{-1}$ y $45.0 \mathrm{mg} \mathrm{l}^{-1}$ (Rubio Arias et al., 


\section{IIVESTIGAGIÓก Y CIECEIA DE LA UחIVERSIDAD AUTÓกOMA DE RGUASCALIERTES}

Figura 8. En el estudio realizado se encontró que el agua es apropiada, en general, para el florecimiento de la vida acuática y del ecosistema en su conjunto. Fotografía tomada por el equipo de investigación.

2004; Holguín et al., 2006) e incluso niveles de 106.33 $\mathrm{mg} \mathrm{l}^{-1}$ en el río Chuvíscar, el cual recibe descargas urbanas de la ciudad de Chihuahua. Al igual que para Mg, no existen restricciones para los niveles de $M n$ incluyendo el agua potable, por lo cual se garantiza su utilización en cualquier proceso. La Organización Mundial de la Salud (OMS) establece que valores superiores a $0.4 \mathrm{mg} \mathrm{l}^{-1}$ podrían ocasionar problemas de mortalidad en niños (Beckman et al., 1985); sin embargo, es importante mencionar que el agua de esta zona no se utiliza como agua potable.

Otros estudios en la misma cuenca han encontrado niveles superiores a los del presente trabajo (Holguín et al., 2006; Rubio Arias et al., 2010).

La presencia de Na en agua ha sido relacionada con descargas agrícolas (Orzepowski y Polikowski, 2008), con la estructura litológica (O'Connor et al., 2010) y el material geológico (Gutiérrez y Carreón Hernández, 2004). La mayor concentración en VZ y en SFC puede ser explicada por el hecho de que este ion se encuentra formando sales disueltas $y$ una vez que el agua es captada, en este caso en la presa, se presenta un efecto de precipitación. Con referencia a los niveles de $\mathrm{Pb}$ aquí reportados, éstos concuerdan con los obtenidos por otros investigadores quienes desarrollaron trabajos en la misma cuenca (Gutiérrez et al., 2008). Los niveles de Se no superaron lo establecido en normas nacionales e internacionales, para riego agrícola que es de $0.02 \mathrm{mg} \mathrm{l}^{-1}$ (NOM-CCA/032-ECOL/1993), por lo cual se infiere que el agua puede ser utilizada para estos fines.

CONCLUSIONES

Se concluye que existen diferencias estadísticas para la calidad del agua en las tres localidades respecto a las variables de T, CE, SDT; así como en la concentración de los metales Al, B, Ca, Cr y Mg. No obstante, y derivado de los niveles de las variables evaluadas, se concluye que el agua es apropiada, en general, para el florecimiento de la vida acuática y del ecosistema en su conjunto. Se recomienda monitorear y vigilar la concentración de elementos que fueron considerados altos en este estudio, una manera de estrategia preventiva, como sería el caso del Al.

LITERATURA CITADA

- ARBAT BOFILL, M. et al. Hydrodynamics of Ribarroja Reservoir (Ebro River, Spain): Water temperature, water velocities and water age. In A. J. Schleiss et al. (Eds.), River Flow 2014 (pp. 1737-1744). CRC Press, 424 pp., 2014.

- BECKMAN, R. A. et al. On the fidelity of DNA replication, manganese mutagenesis in vitro. Biochemistry, 24(21): 5810$5817,1985$.

- BROOKS, K. N. et al. Hidrology and Management of Watersheds. 3a. ed. lowa, US: Blackwell Publishing, 2003.

- CARUSO, B. S. Temporal and spatial patterns of extreme low flows and effects on stream ecosystem in Otago, New Zealand. Journal of Hydrology, 257, 115-133, 2002.

- cCRem lCANAdian council of resource AND ENVIRONMENT MINISTERS). Canadian Water Quality Guidelines. Informe preparado por Task Force on Water Quality Guidelines, 1987.

- CHAREF, A. et al. Irrigation water qualities-Soil pollution (heavy metals and salinity). In Mornag irrigation permiter (SW Tunis, North Tunisia). In Fifteenth International Water Technology Conference. IWTC-15, 2011, Alexandria, Egypt, 2011. 
- CNA (COMISIÓN NACIONAL DEL AGUA). Estadísticas del agua en México, edición 2010. 10 años de presentar el agua en cifras. D. F., México: SEMARNAT, marzo 2010.

- COlegiO DE CHIHUAHUA (2007). El agua en Chihuahua; un ejemplo de mala gestión. Chihuahua, Chihuahua, México: Colegio de Chihuahua, Estudios ambientales, A. C., octubre de 2007.

- DEAN, D. J. y SCHMIDT, J. C. The role of feedback mechanisms in historic channel changes of the lower Rio Grande in the Big Bend Region. Geomorphology, 126, 333-349, 2011.

- GLEICK, P. H. et al. The World's Water 2002-2003: The biennial report on freshwater resources. Washington, US: Island Press, 334 pp., 2002.

- GUTIÉRREZ, M. y BORREGO, P. Water quality assessment of the Rio Conchos, Chihuahua, Mexico. Environment International, 25(5): 573-583, 1999.

- GUtIÉRREZ, M. y CARREÓN HERNÁNDEZ, E. Salinidad en el bajo Río Conchos: aportes y tendencias. Terra Latinoamericana, 22(4): 499-506, 2004.

- GUTIÉRREZ, R. et al. Heavy metals in water of the San Pedro River in Chihuahua, Mexico and its potential health risk. International Journal of Environmental Research and Public Health, 5(2): $91-$ 98, 2008.

- HERNÁNDEZ, G. et al. Evaluación de la contaminación por metales pesados en agua y sedimento de la presa La Boquilla; Fco. I. Madero y Luis L. León, Chihuahua, durante cuatro temporadas climáticas. Memorias. VI Congreso Internacional y XII Congreso Nacional de Ciencias Ambientales. Universidad Autónoma de Chihuahua. Chihuahua, México, 6, 7 y 8 de junio de 2007.

- HOlguín, L. C. et al. Calidad del agua del río Conchos en la región de Ojinaga, Chihuahua: parámetros fisicoquímicos, metales y metaloides. Universidad y Ciencia, 22(1): 51-63, 2006.

- INEGI (INSTItUTO NACIONAL DE ESTADístiCA, GEOGRAFía E INFORMÁTICA). Anuario Estadístico del estado de Chihuahua. México: Autor, 1998.

- INEGI (INSTITUTO NACIONAL DE ESTADÍStICA, GEOGRAFÍA E INFORMÁTICA). Síntesis de Información Geográfica del estado de Chihuahua. Conteo 2005. México: Autor, 2005.

- INEGI (INSTITUTO NACIONAL DE ESTADÍstiCA, GEOGRAFÍA E INFORMÁTICA). Síntesis de Información Geográfica del estado de Chihuahua. México: Autor, 2003.

- KELLY, M. E. El Río Conchos: un informe preliminar. Austin, Texas, US: Texas Center for Policy Studies, 34 pp., 2001.
- Melgar, V. C. E. et al. Efecto de microorganismos con potencial probiótico en la calidad del agua y el crecimiento de camarón Litopenaeus vannamei (Decapoda: Penaeidae) en cultivo intensivo. Revista de Biología Tropical, 61 (3): 12151228, 2013.

- MIYAMOTO, S. et al. Flow of salts and trace elements in the Rio Grande System. College Station, TX, US: Texas Agriculture Experimental Station-Texas Water Resources Institute, 1995.

- MOSLEY, L. M. Drought impacts on the water quality of freshwater systems; review and integration. Earth Science Reviews, 140, 203-214, 2015.

- O'CONNOR, M. et al. Multi-element analysis and geochemical spatial trends of groundwater in rural northern New York. Water, 2, 217-238, 2010.

- ORZEPOWSKI, W. y PULIKOWSKI, K. Magnesium, Calcium, Potassium and Sodium content in groundwater and surface water in arable lands in the commune (Gmina) of Katy Wroclawskie. J. Elementol., 13(4): 605-614, 2008.

- RUBIO, A. H. O. y JIMÉNEZ, J. A. Estadística aplicada con análisis en Minitab. Chihuahua, Chihuahua, México: Editorial de la Universidad Autónoma de Chihuahua y Sindicato de Maestros de la Universidad, abril de 2012.

- RUBIO ARIAS, H. et al. An Overall Water Quality Index (WQI) for a Man-Made Aquatic Reservoir in Mexico. International Journal of Environmental Research and Public Health, 9(5): 1687-1698, 2012.

- RUBIO ARIAS, $\mathrm{H}$. et al. Coliform and Metal Contamination in Lago de Colina, a Recreational Water Body in Chihuahua State, Mexico. International Journal of Environmental Research and Public Health, 8(6): 2386-2400, 2011.

- RUBIO ARIAS, $\mathrm{H}$. et al. Development of a water quality index (WQI) of an artificial aquatic ecosystem in Mexico. Journal of Environmental Protection, 4, 1296-1306, 2013.

- RUBIO ARIAS, H. et al. Metal contamination of a riparian area in the Conchos watershed in Chihuahua, México. Water Resources Management III. Algarve, Portugal, April 11-13, 2005. WIT Transactions on Ecology and the Environment, 80, 269-275, 2005.

- RUBIO ARIAS, H. et al. Water Pollution in the Rio Conchos of Northem Mexico. In G. Latini et al. (Eds.), Development and Application of Computer Techniques to Environmental Studies $X$ (pp. 167-176). UK: WitPress, 2004.

- sansawAT, A. y thIRABUnYANON, M. Anti-aeromonas hydrophila activity and characterisation of novel probiotic strains of Bacillus subtilis isolated from the gastrointestinal tract of giants freshwater prawns. Maejo International Journal of Science and Technology, 3(1): 77-87, 2009. 
- sedesol (SeCRetaría de desarRollo social). Norma Mexicana CCA/032-ECOL/1993, que establece los límites máximos permisibles de contaminantes en las aguas residuales de origen urbano o municipal para su disposición como riego agrícola. Diario Oficial de la Federación, 18 de octubre de 1993.

- SEDUE (SECRETARÍA DE DESARROlLO URBANO y ECOLOGÍA). Criterios ecológicos de calidad de agua CE-CCA-001/89SEDUE. Diario Oficial de la Federación, 13 de diciembre de 1989.

- tagliabue, M. et al. Boron removal from water: needs, challenges and perspectives. Journal of Cleaner Production (número especial titulado Emerging Industrial Processes for Water Management), 56-64, 2014.

- WEBB, B. W. et al. Recent advances in stream and river temperature research. Hydrological Processes, 22 (7): 902-918, 2008.

\section{De páginas electrónicas}

- KOCHKODAN, V. et al. The chemistry of boron in water. 2. Boron Separation Processes (pp. 35-63). Elsevier, 2015. doi: 10.1016/ B978-0-444-63454-2.00002-2

- $\quad$ NIH (NATIONAL INStitute OF HEALTH). Office of Dietary Supplements. Health Information. 2013. Recuperado el 21 de marzo de 2013, de http://ods.od.nih.gov/factsheets/calciumconsumer

- ONU (ORGANIZACIÓN DE LAS NACIONES UNIDAS). Declaración Universal de los Derechos Humanos. Recuperado de http:// www.un.org/es/universal-declaration-human-rights/

- PREeCE, R. M. y JONES, H. A. The effect of keepit dam on the temperatura regime of the Namoi River, Australia. River Research and Applications, 18(4): 397-414, 2002. doi: 10.1002/ rra.686

- RUBIO ARIAS, H. et al. Contamination of the Conchos River in Mexico: Does it pose a health risk to local residents?
International Journal of Environmental Research and Public Health, 7(5): 2071-2084, 2010. doi: 10.3390/ijerph7052071

- SCFI (SECRETARÍA DE COMERCIO Y FOMENTO INDUSTRIAL). NMX-AA-007-SCFI-2000. Análisis de agua-Determinación de la temperature en aguas naturales, residuals y residuals tratadas-Método de prueba (cancela a la NMX-AA-007-1980). Recuperado de http://biblioteca.semarnat.gob.mx/janium/ Documentos/Ciga/agenda/DOFsr/NMX-AA-007-SCFI-2000.pdf

- SCFI (SECRETARÍA DE COMERCIO Y FOMENTO INDUSTRIAL). NMX-AA-014-1980. Norma Mexicana. Cuerpos receptores. Muestreo. México, D. F., 1980. Recuperado de http://lasa. ciga.unam.mx/monitoreo/images/biblioteca/14\%20NMXAA-014-1980\%20Cuerpos\%20receptores-Muestreo.pdf

- SCFI (SECRETARÍA DE COMERCIO Y FOMENTO INDUSTRIAL). NMX-AA-008-SCFI-2000. Análisis de agua-Determinación del pH-Método de prueba (cancela a la NMX-AA-008-1980). Recuperado de http://www.agua.org.mx/bibliotecatematica/marco-juridico-del-agua/1466-normasmexicanas/15150-nmx-aa-008-scfi-2000-analisis-de-aguadeterminacion-del-ph-metodo-de-prueba-

- SCFI (SECRETARÍA DE COMERCIO Y FOMENTO INDUSTRIAL). NMX-AA-051-SCFI-2001. Análisis de agua-Determinación de metales por absorción atómica en aguas naturales, potables, residuales y residuales tratadas-Método de prueba (cancela a la NMX-AA-051-1981). Recuperado de http://www.agua. org.mx/biblioteca-tematica/marco-juridico-del-agua/1466normas-mexicanas/15242-nmx-aa-051-scfi-2001-analisis-deagua-determinacion-de-metales-por-absorcion-atomica-enaguas-naturales-potables-residuales-y-residuales-tratadasmetodo-de-prueba

- SCFI (SECRETARÍA DE COMERCIO Y FOMENTO INDUSTRIAL). NMX-AA-093-SCFI-2000. Análisis de agua-Determinación de la conductividad electrolítica-Método de prueba (cancela a la NMX-AA-093-1984). Recuperado de http://www.agua.org.mx/ biblioteca-tematica/marco-juridico-del-agua/1 466-normasmexicanas/15321-nmx-aa-093-scfi-2000-analisis-de-aguadeterminacion-de-la-conductividad-electrolitica-metodo-deprueba 